\title{
ADVANCED DEFORMATION STAGES IN DUPLEX STEEL INVESTIGATED USING NEUTRON AND SYNCHROTRON RADIATION
}

\author{
E. Gadalińska ${ }^{1}$, A. Baczmański ${ }^{2}$, Y. Zhao ${ }^{3}$, L. Le Joncour ${ }^{3}$, S. Wroński $^{2}$, B. Panicaud ${ }^{3}$,

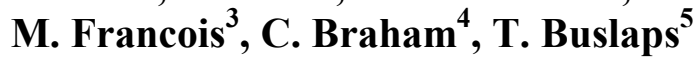 \\ ${ }^{1}$ Institute of Aviation, Materials and Structures Research Center, \\ Al. Krakowska 110/114, 02-256 Warsaw, Poland \\ ${ }^{2}$ AGH-University of Science and Technology, Faculty of Physics and Applied Computer Science, \\ Al. Mickiewicza 30, 30-059 Kraków, Poland \\ ${ }^{3}$ ICD-LASMIS, Université de Technologie de Troyes (UTT), UMR CNRS 6281, \\ rue Marie Curie, CS 4206, 10004 Troyes, France \\ ${ }^{4}$ PIMM, Arts et Métiers ParisTech (ENSAM), 151 Bd de l'Hôpital 75013 Paris, France \\ ${ }^{5}$ ESRF, 6 rue J. Horowitz, 38500 Grenoble Cedex, France
}

$\underline{\text { elzbieta.gadalinska@ilot.edu.pl }}$

\begin{abstract}
The grain scale of materials is an area still open for investigations within the field of materials science. The most helpful tools to perform this type of research are diffraction methods. Within the research project presented in this paper two experiments were carried out employing two different types of radiation: neutron (ISIS) and synchrotron (ESRF). The aim of the work was to describe the stress state in the necking zone during the occurrence of a damage phenomenon (Fig. 1.) in separate phase and to check the level of the homogeneity. The supplemental tools were the finite elements method and self-consistent modeling - it testified, confirmed and completed our experimental results and allowed us to formulate the justifiable conclusions.
\end{abstract}

Keywords: Two-Phases materials, Mechanical behaviour, Diffraction methods.

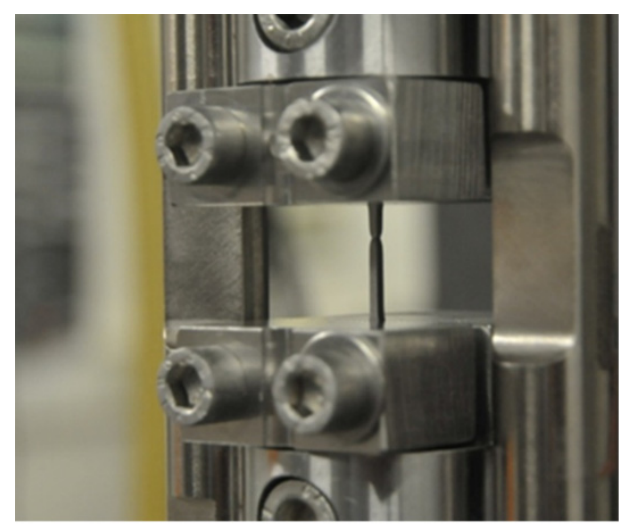

a)

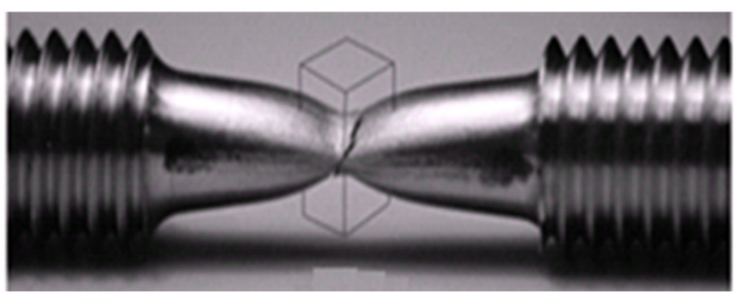

b)

Fig. 1. a) The specimen in the tensile machine rig during the experiment with synchrotron radiation. The necking area visible and b) the broken specimen after the tensile test performed with neutron radiation. 


\section{EXPERIMENT}

\section{Material}

The most appropriate material for the experiment was duplex stainless steel UR45N, which is composed of two phases: ferrite and austenite in equal proportions $(50 \%$ of ferrite and $50 \%$ of austenite). The production process of UR45N steel consists of the following steps: continuous casting, hot rolling to form a steel sheet, thermal aging at a work temperature of $400^{\circ} \mathrm{C}$ for 1000 hours and, finally, cooling down at room temperature. The above described process induced a strong texture (Fig. 2: the austenite islets are visible as spreaded in ferrite). The chemical composition of this steel is presented in Table 1. During the in-situ tensile test the sample was loaded in the direction of the rolling direction of the steel sheet.

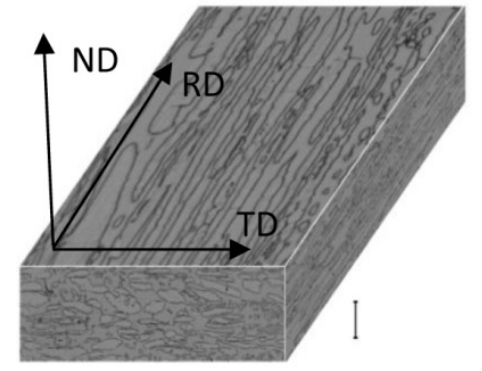

a)

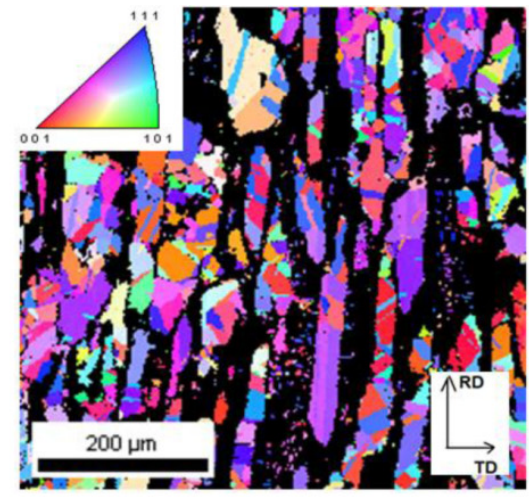

b)

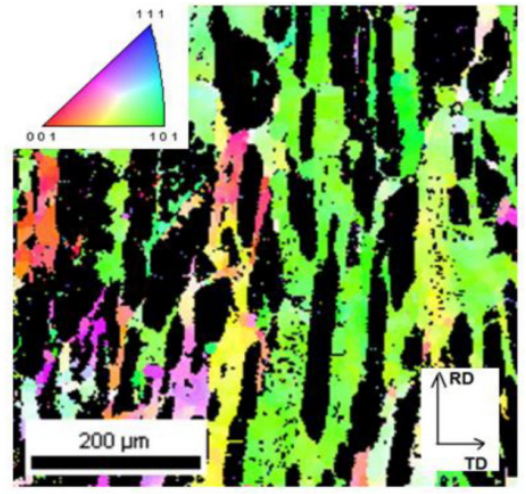

c)

Fig. 2. a) The RD, ND, TD directions' definition and the structure of the duplex stainless steel b) with austenite islets (purple and violet) in c) ferrite (green).

Table 1. The chemical composition of UR45N duplex steel.

\begin{tabular}{rrrrr}
\hline \multirow{2}{*}{ UR45N } & $\mathbf{C}$ & Mn & $\mathbf{C r}$ & Ni \\
\cline { 2 - 5 } & 0.015 & 1.6 & 22.4 & 5.4 \\
& Mo & $\mathbf{C u}$ & $\mathbf{S}$ & $\mathbf{N}$ \\
\cline { 2 - 5 } & 2.9 & 0.12 & 0.001 & 0.17 \\
\hline
\end{tabular}

\section{Specimens}

Two types of specimens were prepared for the experiments. The neutron diffraction experiment was performed on cylindrical specimens whose measurement area was $14 \mathrm{~mm}$ length and $8 \mathrm{~mm}$ diameter (Fig. 3a). The specimens for the synchrotron experiment were dog-bone shaped with an effective length of $10 \mathrm{~mm}$ and a cross-section $1,5 \times 1,5 \mathrm{~mm}^{2}$ (Figs. 3b-d). In both cases, the loading direction was the same as the rolling direction (RD). In the case of the synchrotron experiment, 2 mesurements were made with 2 specimens of different orientations: for the first specimen, the transverse direction was parallel to the incident beam and for the second one, the normal direction was parallel to the incident beam. 
Neutron diffraction

Engine-X on ISIS

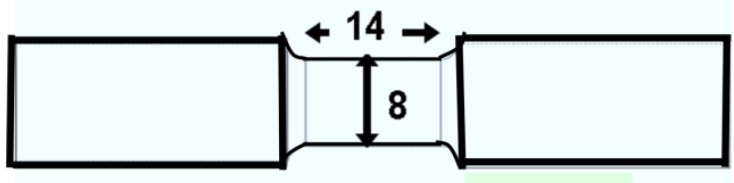

Threads M12

Loading/Rolling direction (RD)

a)

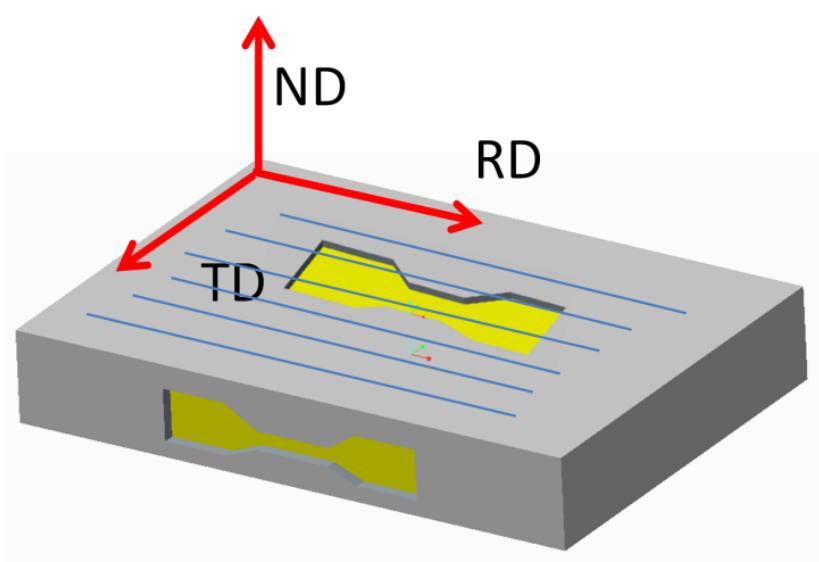

c)
Synchrotron difraction

ID15B beamline on ESRF

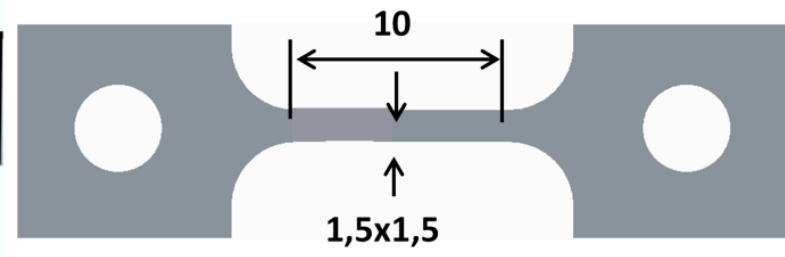

Loading/Rolling direction (RD)

b)

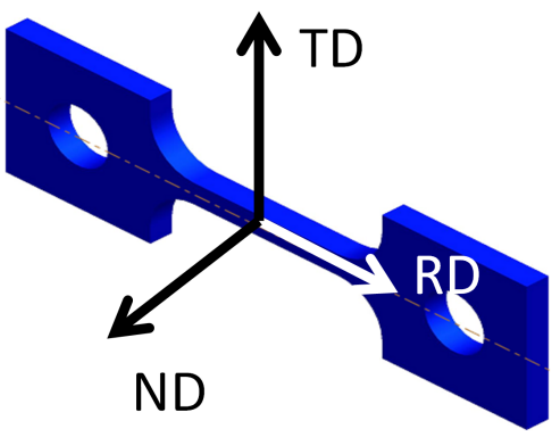

d)

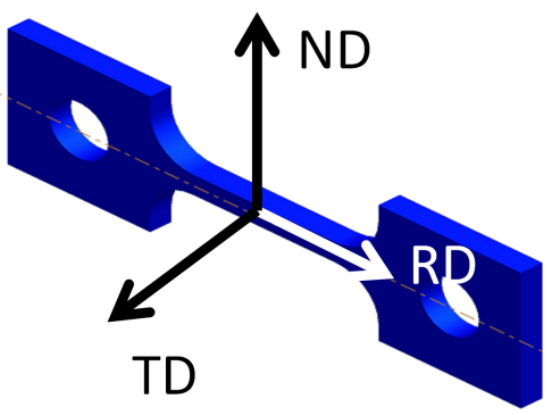

e)

Fig. 3. Specimens: a) for neutron diffraction experiment (NDE); b) for synchrotron diffraction experiment (SDE), c) and d) directions in the NDE specimens.

\section{Calibration}

The crucial point in our investigations was to introduce and apply a reliable calibration procedure that would enable the estimation of the real value of stress for high loading values, when the necking phenomenon appears, e.g. when the cross-section of the specimen starts to narrow every loading step (before that happens the specimen can be considered as homogenously deformed). When the heterogeneous deformation appears the real stress values cannot be calculated according to the equation (1) due to the evolution of the cross-section of the specimen which is unknown. When the relation described by equation 1 is applied to the necking phenomenon the relation $\left\langle\varepsilon_{R D}\right\rangle_{\{h k l\}}$ vs. $\sum_{R D}^{\text {nominal }}$ cannot be considered as correct (Figs. 4a-b).

$$
\sum_{R D}^{\text {nominal }}=\frac{F}{S_{0}}
$$




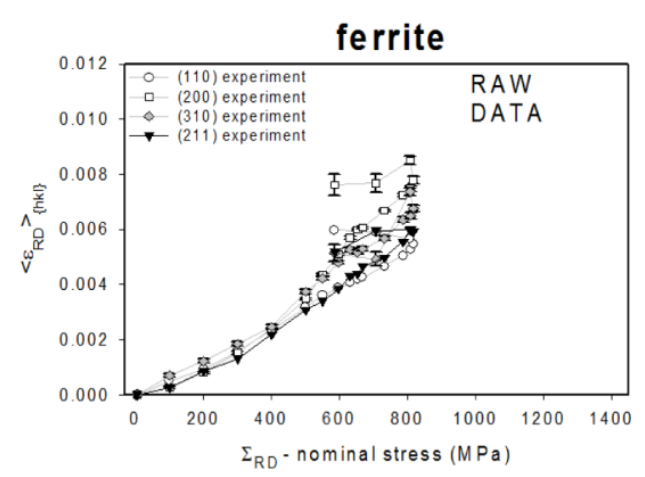

a)

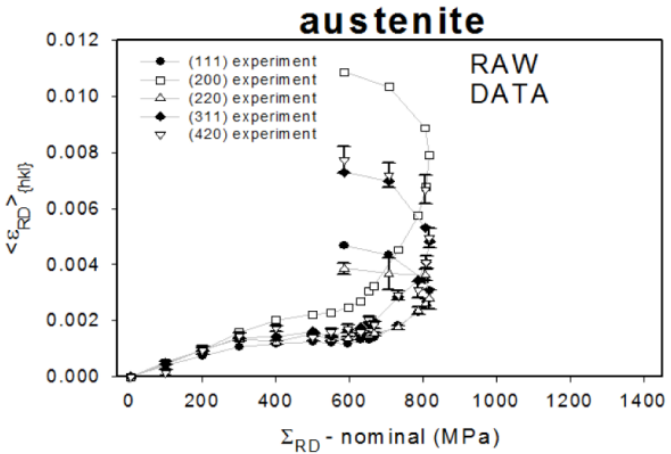

b)

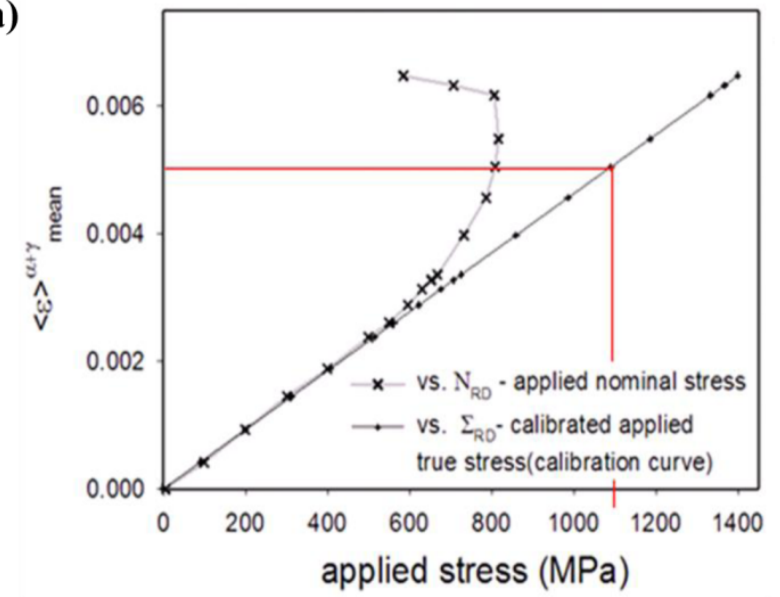

c)

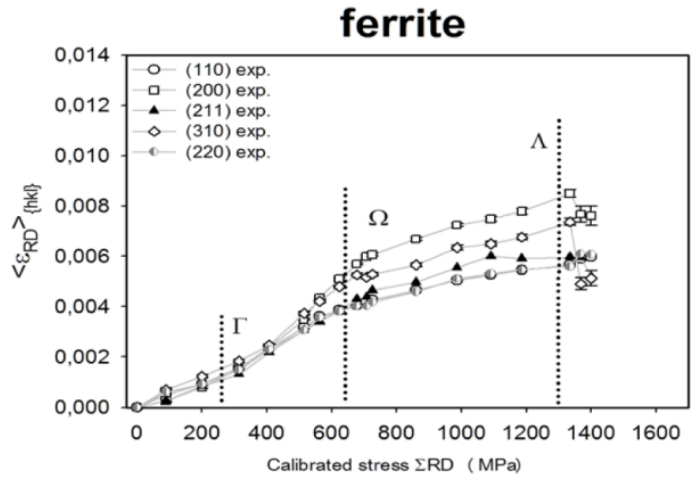

d)

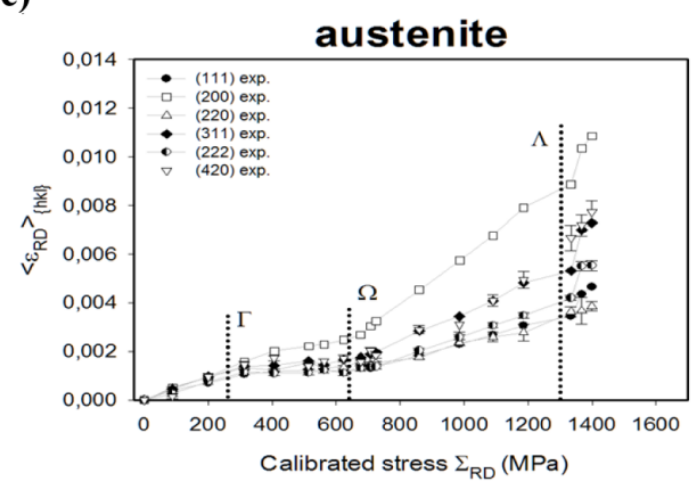

e)

Fig. 4. The calibration procedure. The strain vs. stress plots for nominal macrostress values a) for ferrite phase and b) for austenite phase; c) calibration idea; the strain vs. stress plots for calibrated macrostress values d) for ferrite and e) for austenite.

The calibration procedure for cases of this type was worked out by Baczmański in [15] and applied in this work. In Figure 4c, two plots are presented: the plot showing relations between average strain for all $h k l$ 's and both phases $\langle\varepsilon\rangle_{\text {mean }}^{\alpha+\gamma}$ vs. applied nominal stress $\sum_{R D}^{\text {nominal }}$ and vs. the calibrated applied true stress $\sum_{R D}^{\text {calibrated }}$. The value of $\langle\varepsilon\rangle_{\text {mean }}^{\alpha+\gamma}$ was calculated according to Eq. (2) and the calibrated macrostress according to Eq. (3) where $k$ is a scalar coefficient describing the relationship between macrostress in diffraction gauge volume and the arithmetic mean calculated over all available lattice strains measured for both phases. 


$$
\begin{gathered}
\langle\varepsilon\rangle_{\text {mean }}^{\alpha+\gamma}=\frac{\left\langle d_{R D}^{\Sigma}\right\rangle_{\{h k l\}}-\left\langle d_{R D}^{0}\right\rangle}{\left\langle d_{R D}^{0}\right\rangle_{\{h k l\}}} \\
\sum_{R D}^{\text {calibrated }}=k\left\langle\varepsilon_{R D}\right\rangle_{\text {mean }}
\end{gathered}
$$

The main assumption of the calibration procedure was that the relation between overall stress and strain is proportional for the whole range of deformation: elasto-plastic stage, large deformation stage even in damage stage (Fig. 4c). This assumption allowed us to calculate the $k$ coefficient and, in effect, estimate the true value of stress in stages of large deformation and damage phenomenon. The reliable $\left\langle\varepsilon_{R D}\right\rangle_{\{h k l\}}$ vs. $\sum_{R D}^{\text {calibrated }}$ plots are presented in Figs. 4d-e.

\section{Diffraction measurements setups}

To achieve the objectives of the work two experiments were performed applying different types of radiation. The first experiment was performed at a neutron source at the ISIS Rutherford Appleton laboratory, in Oxford, United Kingdom. The second experiment was performed at the ESRF, Grenoble, France, where the synchrotron radiation was applied. In both cases the in-situ tensile test was performed.

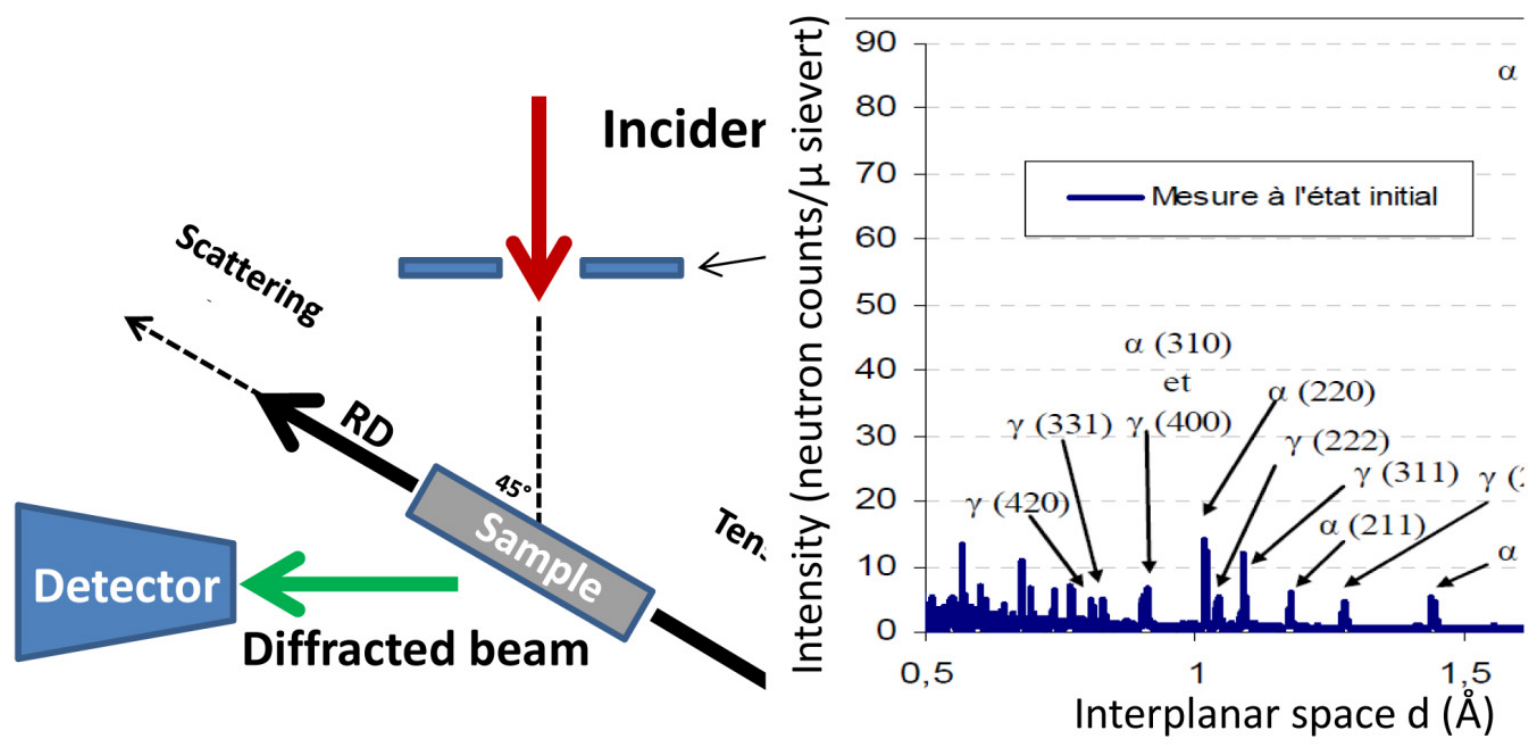

a)

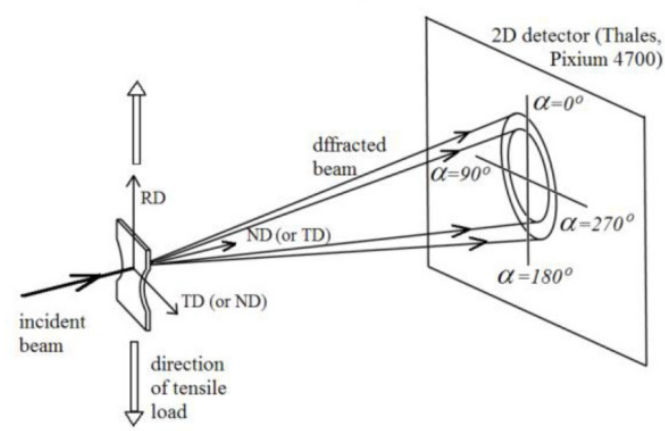

c) b)

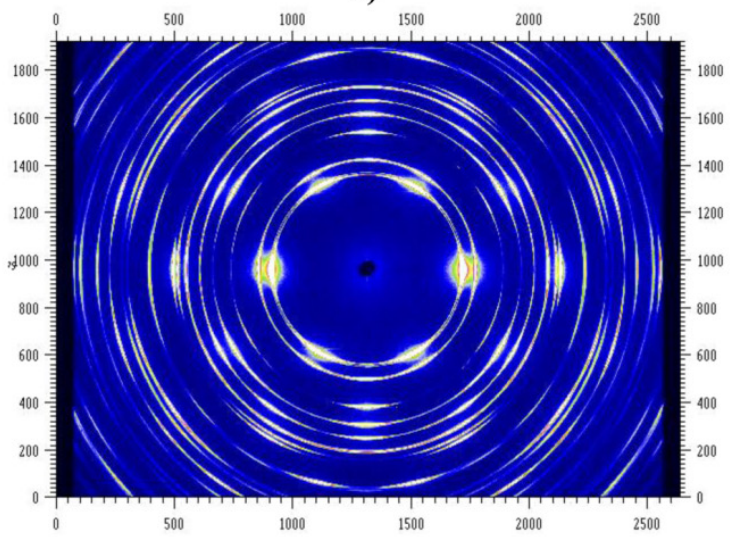

d)

Fig. 5. Experimental geometries and diffractograms for a)-b) neutron diffraction experiment and c)-d) for synchrotron radiation experiment. 
In Figs. 5a-b, the geometry of the experiment in time-of-flight mode is presented including the resulting diffratogram. The specimen was subjected to the tensile loading along its axis (rolling direction, RD) and simultaneously irritated by the neutron beam at an angle of 45 degrees. It was diffracted at the angle $2 \theta=90^{\circ}$ and received by the detector. The specificity of the time-of-flight measurement mode is that the neutrons of different wavelengths are emitted by the nuetron source and that's why we can obtain the information about different lattice distances simultaneously (for different phases and $h k l$ 's directions). In the case of this experiment the examined gauge was $4 \mathrm{~mm} \times 4 \mathrm{~mm}$.

The idea of the synchrotron experiment is presented in Figs. 5c-d. The experiment was performed in transmission mode (monochromatic radiation with the wavelength $\lambda=0,14256 \AA$ ). The diffracted beam was aquisited by the 2D detector Thales Pixium 4700 (there was also another detector, Pico1, measuring the loss of the intensity after passing though the specimen). The onedimensional diffractograms were obtained by integration of 2D images with the Fit2D software. The beam size for this experiment was $100 \mu \mathrm{m} \times 100 \mu \mathrm{m}$ for "dog-bone" shape specimens whose cross section was $1,5 \mathrm{~mm} \times 1,5 \mathrm{~mm}$. The specimens of two types were subjected to the experiment: the "ND" and "TD" specimens. The "ND" specimen was manufactured so that the incident X-ray beam was parallel to ND direction (Fig. 3d); analogously for "TD" specimen (Fig. 3e). The load was applied along the RD for both samples.

\section{RESULTS ANALYSIS}

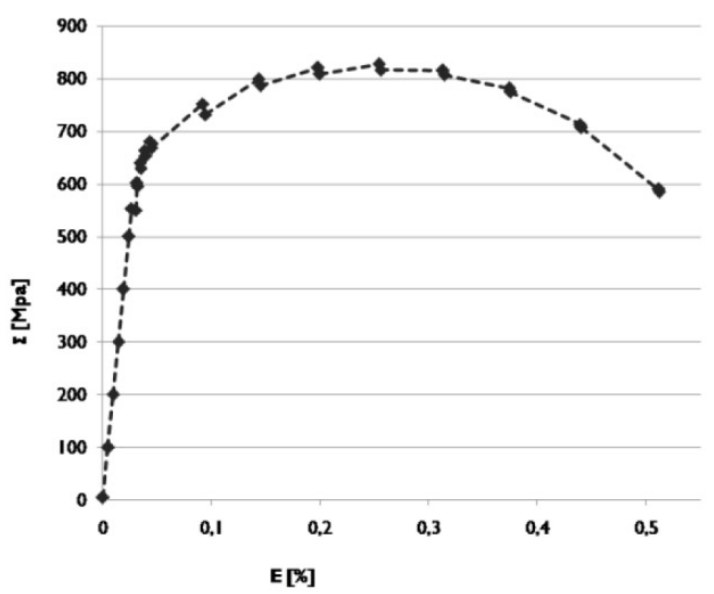

a)

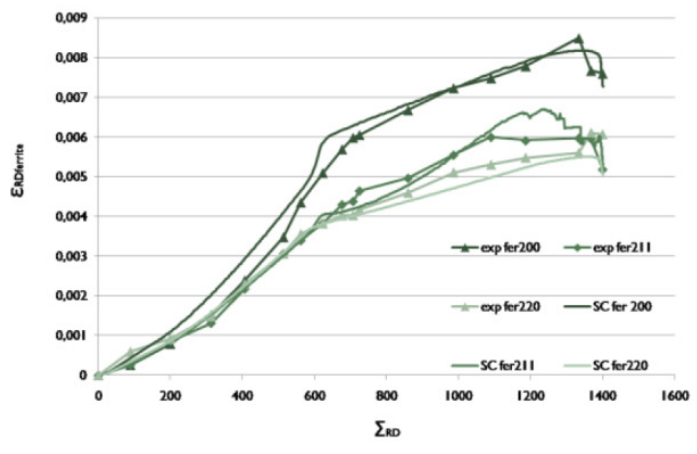

c)

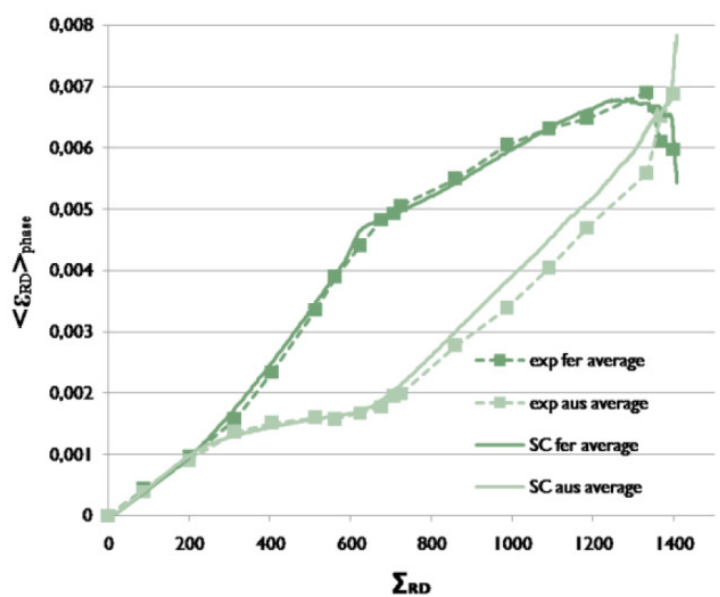

b)

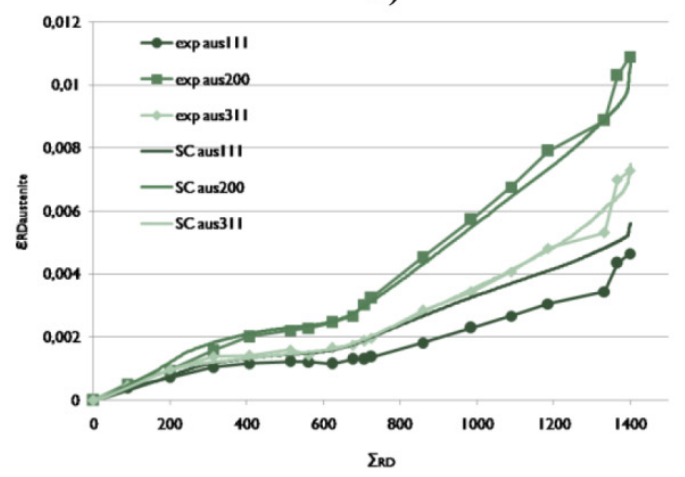

d)

Fig. 6. Comparison of experimental data (points and lines) and self-consistent modeling (lines) for neutron diffraction experiment: a) macroscopic curve, b) the dependence of average strain for separate phases versus macrostress, $c$ ) the strain values for different reflections for ferrite and d) for austenite. Both values in direction of tensile forces applied during experiment (RD direction). 
Neutron diffraction experiment results - macro/mesoscopic damage process modeling.

The values of strains for every stage of deformation for both phases presented in Figures 6a-d include the results of the experiment as well as the modeling outcomes. The model calculations were based on 2000 grains while for the initial texture the ODF function was measured and used as input information. For ferrite, we assumed two slip systems: $\langle 111\rangle\{211\} ;\langle 111\rangle\{110\}$; the hardening parameter value was $H=110 M P a$, the initial critical shear stress was $\tau_{c r}=350 M P a$ and the measured value of initial stress was $\sigma_{0 F e}=-57 M P a$. For austenite only one slip system was initiated: ?10 10 (111\}, and above values were: $H=225 \mathrm{MPa}, \tau_{c r}=140 \mathrm{MPa}$ and $\sigma_{0 \text { Aust }}=87 \mathrm{MPa}$ respectively.

Plotted below (Figs. 6a-d) are 4 stages of deformation. The first stage when both phases e.g. ferrite and austenite are in the elastic region, the second stage when the plastic deformation of austenite appears, the third stage when the ferrite phase goes to the plastic region and the last, fourth stage - the stage of damage where a rapid decrease of the ferrite phase takes place (probably, the fracture appears in this phase) and the rise of strain in the austenitic grains. The identification of ductile damage in ferrite allows us to put damage parameters only for ferrite phase.

The presented above investigation allows us to indentify four mechanical behaviour stages. The good coherence between experimental data and modeling of both phases and of all individual $h \mathrm{kl}$ planes is observed.

\section{Synchrotron diffraction experimental results \\ Macro/mesoscopic damage process}

In the neutron diffraction experiment, the lattice strains were measured during the tensile test performed applying an external load of the required value e.g. the experiment was performed in the load control mode. In the load control mode an immediate fracture can occur due to the concentration of stress in the deformation neck and due to the instability of deformation. The main difference between the neutron and synchrotron experiments was that the latter one was performed in a strain control mode, which allowed the deformation in the neck to be observed more precisely. When the strain control mode is applied, during a progressively increasing sample strain, the loading of the sample decreases, preventing the concentration of the large strain in the narrowest section of the neck. The plots presenting the relation between stress and strain (Figs. 7c-d) was prepared for true values i.e. for values which were recalculated when the narrowing of the neck region was taken into account. The rig elasticity was taken into account as well. The necking phenomenon can be easily observed by the stress reduction appearing at the end of the plots (points A, B, C) in Figures 7a-d. The deformation neck was observed through an optical lunette during measurements. To analyze the data obtained, the initial stress values in different directions with respect to the specimen geometry were taken into account (Table 2). On the macro- and microcurves presented in Figures 7a-d, three points: A, B and C (C') are tabbed. Points A and B describe an early stage of necking and $\mathrm{C} / \mathrm{C}$ ' points design the state just before the fracture of the sample has occured (the damage state). Figures $7 \mathrm{c}-\mathrm{d}$ show that while in early necking stages the strains in ferrite are bigger than those in austenite, at a more advanced damage stage, probably due to cracks appearing in ferrite phase, the strains values in this phase decrease and a bigger part of the load is carried by austenite phase.

Table 2. The values of initial stresses in separate phases of stainless duplex steel.

\begin{tabular}{lcccc}
\hline & & $\boldsymbol{\sigma}_{\mathbf{0 R \boldsymbol { D }}}(\mathrm{MPa})$ & $\boldsymbol{\sigma}_{\mathbf{0 T} \boldsymbol{D}}(\mathrm{MPa})$ & $\boldsymbol{\sigma}_{\mathbf{0 N \boldsymbol { D }}}(\mathrm{MPa})$ \\
\hline TD specimen & Austenite & $134 \pm 15$ & $84 \pm 15$ & $-218 \pm 15$ \\
& Ferrite & $-155 \pm 19$ & $-44 \pm 19$ & $199 \pm 18$ \\
ND specimen & Austenite & $-34 \pm 11$ & not measured & not measured \\
& Ferrite & $59 \pm 19$ & & \\
\hline
\end{tabular}




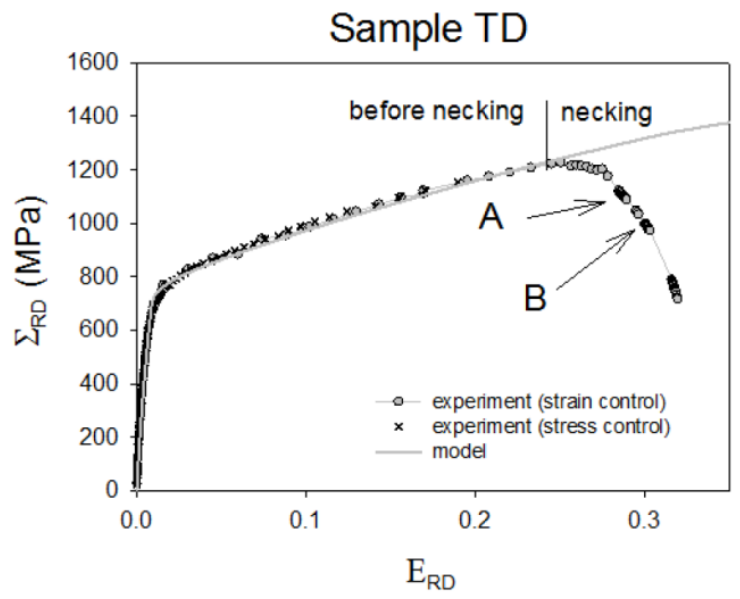

a)

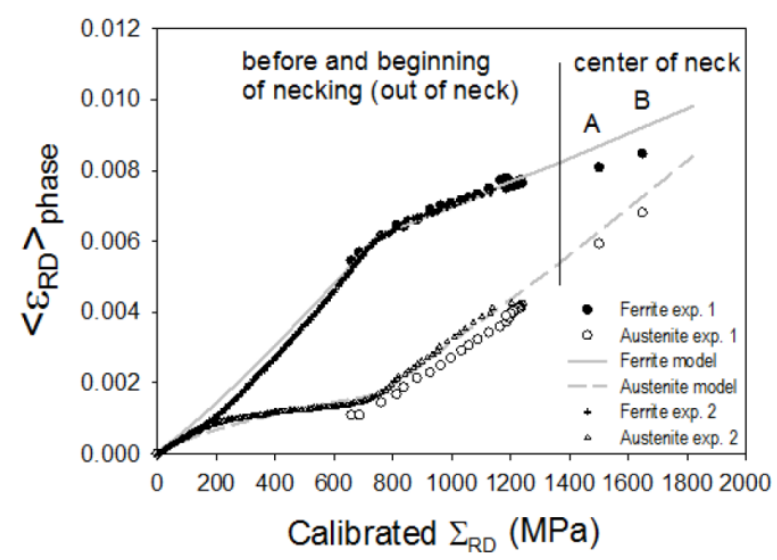

c)

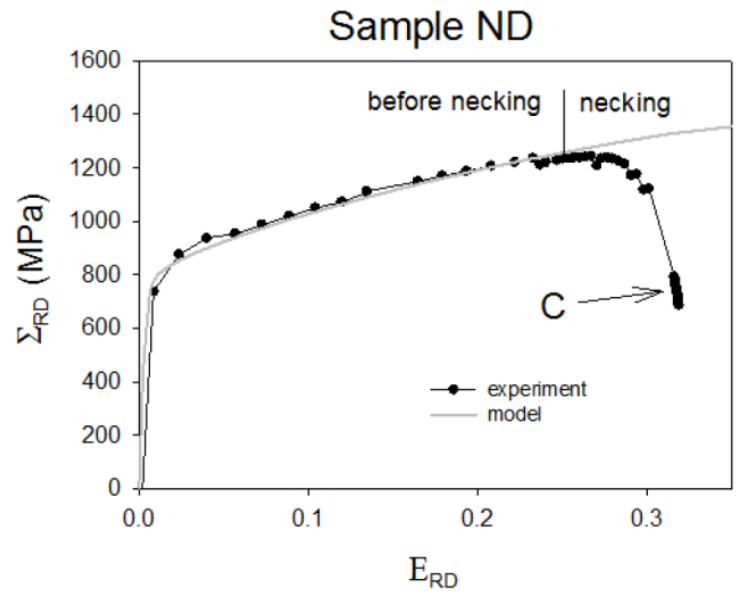

b)

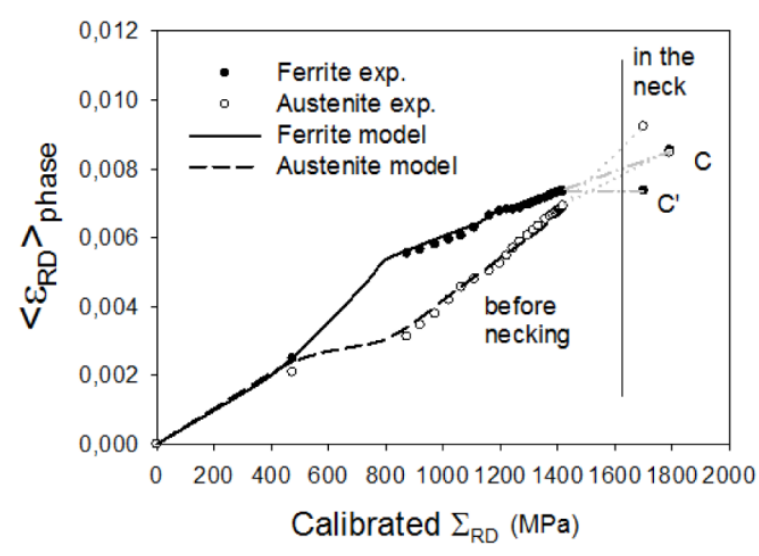

d)

Fig. 7. Macrocurves $\Sigma_{R D} v S . E_{R D}$ for two specimens examined in synchrotron radiation experiment: a) for TD specimen (the incident beam was parallel to the TD direction) and b) ND specimen (respectively). Average strains $\left\langle\varepsilon_{R D}\right\rangle_{\text {phase }}$ versus calibrated macrostress $\Sigma_{R D}$ for separate phases for c) TD specimen and d) for ND specimen. Points $A$ and $B$ designate latterly described scans for not advanced necking process and point $C$ and $C$ '- scans for advanced necking (just before fracture). Points $A, B$, and $C$ are scans for the center of the neck and point $C$ ' is a scan of a position near to the center of the neck.

\section{FEM results}

To obtain the information about the contribution of geometry to the distribution of the stress, the finite element modeling was conducted - mainly focusing on the influence of the neck shape on stress distribution (two-phase structure was not considered in the calculations). The software employed was Abaqus CAE 6.13. The sample dimensions in the necking area were estimated, which allowed us to build a three-dimensional geometric model (Figs. 8a-b). The simulation within the elastic deformation stage used the anisotropic overall elastic constants determined by a self-consistent model; the overall stiffness tensor (Table 3) was calculated from single crystal elastic constants of each phase accounting the crystallographic texture - it was introduced into the model to account for elastic anisotropy of the sample. The time period of the dynamic implicit step was $100 \mathrm{~s}$. The uniform and homogeneous pressure $P=80 \mathrm{MPa}$ was imposed on the two ends of the specimen (Fig. 8a). Two types of mesh sizes were applied to counterbalance the computation speed and the accuracy of the numerical simulation. The mesh with a free structured tetrahedral area was used on the out-necking area (the element seeds were assigned every $0,5 \mathrm{~mm}$ along the edge of the model). The difference in the element applied in the necking area was that the distance 
between the element nodes was reduced to $0,05 \mathrm{~mm}$ in order to get a more accurate result along the necking. Figures 8c-e present the evolution of the simulated principal stresses, assuming that shear stresses were negligible, the principal strain in the direction of the applied load, and the von Mises stress in position function along the neck. The analysis of these plots led to the conclusion that due to the three-axiality of stresses in the necking region the maximum von Mises stress and strain are not concentrated in the narrowest part of the neck, but at same distance from that place.

A very interesting correlation is visible in Figures 8d-e where the macrostrain along RD direction and von Mises stresses are presented. Both plots show a significant decrease in strain/von Mises stresses in the center of the neck; the maximum value is reached at the distance of approximately $0,3 \mathrm{~mm}$ from the centre for macrostrain and at about $0,5 \mathrm{~mm}$ from the centre for von Mises stresses. The conclusion drawn based on these plots is that the equivalent von Mises stress plays an important role in the softening of the ferrite and probably leads to the initiation of the damage process in this phase. In both figures we can observe that the value of the von Mises macrostress increases at some distance from the neck centre. The reason for it is the three-axiality of the macrostress leading to more advanced evolution processes occurring in the phases.

Table 3. Overall stiffness tensor used for finite elements modeling.

\begin{tabular}{ccccccccc}
\hline $\boldsymbol{C}_{\mathbf{1 1}}$ & $\boldsymbol{C}_{\mathbf{2 2}}$ & $\boldsymbol{C}_{\mathbf{3 3}}$ & $\boldsymbol{C}_{\mathbf{1 2}}$ & $\boldsymbol{C}_{\mathbf{1 3}}$ & $\boldsymbol{C}_{\mathbf{2 3}}$ & $\boldsymbol{C}_{\mathbf{4 4}}$ & $\boldsymbol{C}_{\mathbf{5 5}}$ & $\boldsymbol{C}_{\mathbf{6 6}}$ \\
\hline \multicolumn{8}{c}{$[\mathrm{GPa}]$} \\
261 & 265 & 252 & 99 & 112 & 108 & 84 & 88 & 74 \\
\hline
\end{tabular}
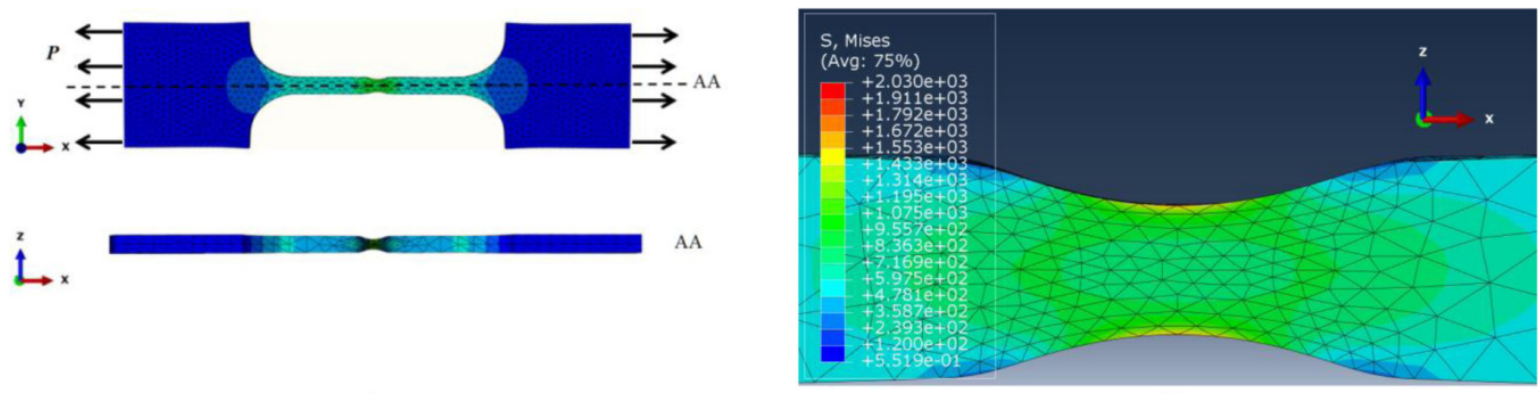

a)

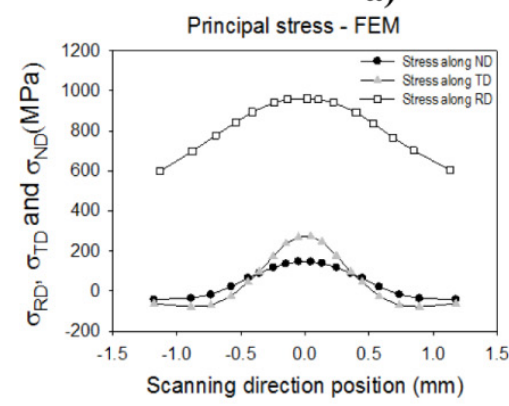

c)

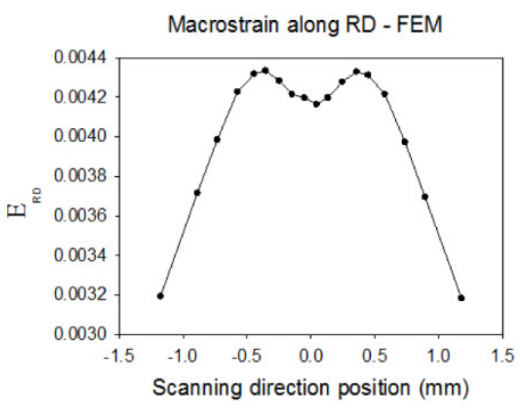

d)

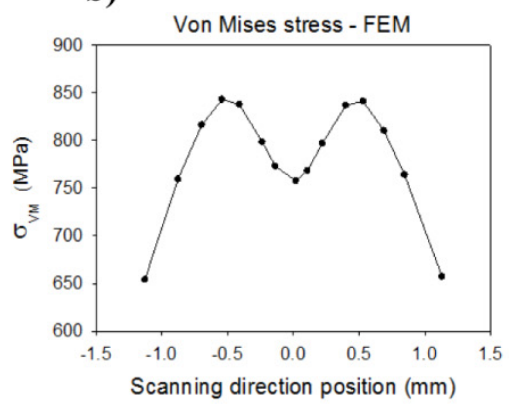

e)

Fig. 8. FE modeling and its results: a) forces and b) meshes applied to the specimen; the results: c) stresses along different directions in the specimen, d) marcostress along RD direction e) von Mises stress. All values versus the position in the neck.

\section{Lattice strain evolution in the necking region}

The evolution of the lattice strains along the neck is described based on the scans obtained along the deformation neck for the stages A, B and C (Figs. 9 and 10). In the case of not advanced necking (points $\mathrm{A}$ and $\mathrm{B}$ ) we can observe bigger values of strain in austenite than those in ferrite 
along the whole length of neck. Comparing the results for points A and B we can observe that in both phases in the centre of the neck the difference between strains decreases faster than for other positions in the neck. This is likely to be caused by the highest stress concentration and the most advanced plastic deformation in this region. Definitely different picture is observed in Figure 9c where the analysis for points $\mathrm{C} / \mathrm{C}^{\prime}$ is presented. In the center of the neck (point $\mathrm{C}$ ) the lattice strain values are approximately equal in both phases while in the symmetrical position on both sides of the neck (point C') a visible inversion of the phase's strains occurs (strain in the austenite is much higher than that in the ferrite). This phenomenon can be explained by the fact that the transition of the load from the ferritic phase to the austenite took place as a result of a possible damage process initiated in the ferrite. These conclusions confirm the neutron diffraction experiment results with a much better spatial resolution.

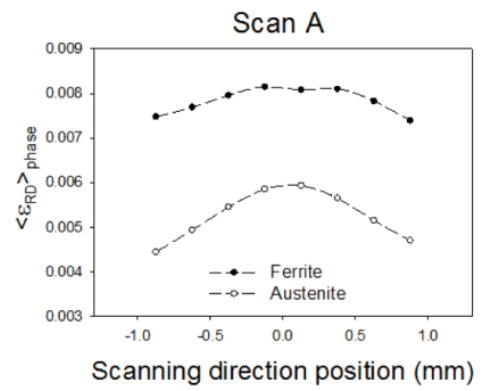

a)

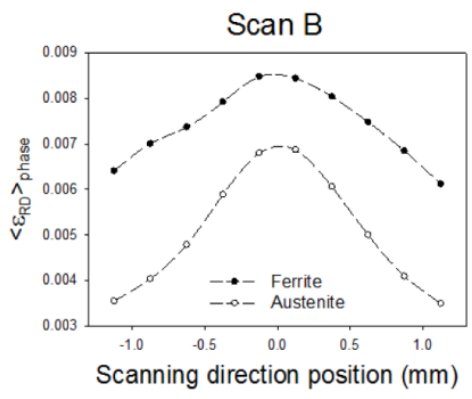

b)

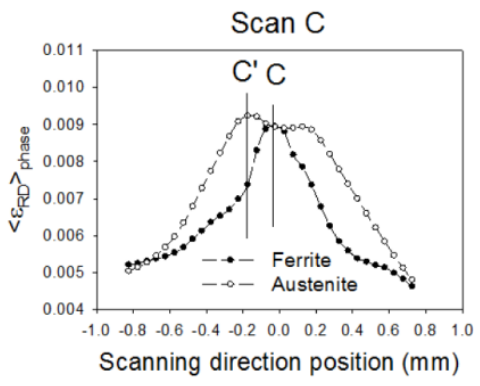

c)

Fig. 9. Synchrotron experiment results for different points on $\Sigma_{R D} v s . E_{R D}$ curves $\left(A, B, C\right.$ and $\left.C^{\prime}\right)$ : plots show average strain values $\left\langle\varepsilon_{R D}\right\rangle$ for separate phase in $R D$ direction versus the position in the neck.

\section{The influence of different reflections}

Figures $10 \mathrm{a}-\mathrm{b}$ show the dependence of lattice strains on separate hkl's in particular phases during the in-situ tensile test across the length of the neck. The 211 and 200 reflections' strain values of ferrite exhibit different dependence on scanning position compared with the calculated von Mises stress and strain. There, the highest measured strain is concentrated in the narrowest part of the neck and significantly decreases with the distance from this region. This effect can be caused by damage occurring in the groups of grains contributing to reflections 211 and 200 in the ferrite.

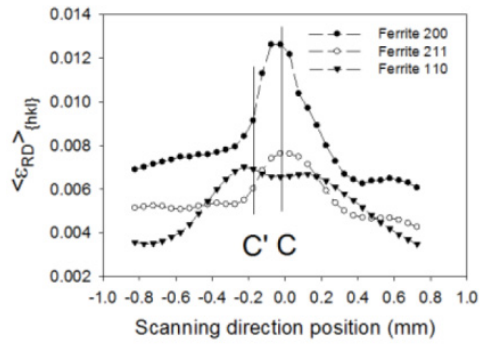

a)

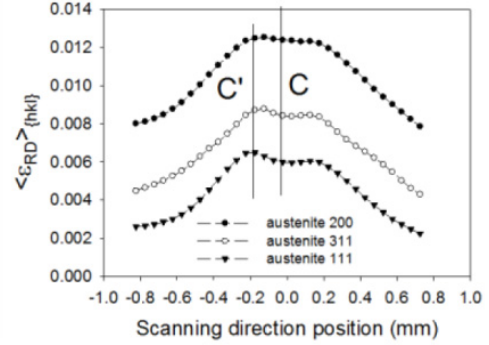

b)

Fig. 10. Average strain values $\left\langle\varepsilon_{R D}\right\rangle$ for points $C$ an $C$ ' on macrocurve for a) ferrite and b) austenite phase for different hkl reflections.

We found out that not all grains in a particular phase are responsible for the strain and stress inversion between phases, which can be observed in Figures 10a-b. For all measured austenite lattice planes, we can observe very similar variation as well as for the ferrite 110 plane. For ferrite 211 and 200 the very sharp decrease of strains with an increasing distance from the center of the neck is observed. This observation confirms the results for neutron measurements form [15] where 
the sharp decrease of lattice strains for 211 and 200 ferrite reflections was observed near to the fracture of the specimen. The character of the strain distribution in 110 reflection was similar as for the case of the synchrotron radiation: it didn't change significantly. The conclusion is that the ferritic grains having orientations which contribute to the 211 and 200 reflections, behave differently than other grains in the sample. Probably the reason is that the softening of the damaged grains leads to a reduction of the localised stresses and cause a transfer of the load to the other grains in the sample (mostly to the austenitic grains). However this process depends on the position in the neck.

\section{CONCLUSIONS}

The aim of this work was to study the mechanical behavior of separate phases of duplex steel during advanced tensile deformation called a necking phenomenon. Firstly, it was concluded that the deformation in the examined steel occurred differently in the two phases: it appeared that yield stress of the ferrite was much higher than that in the austenite. Additionally, we observed that the yield stress value depended highly on initial stresses in the phases. During the results analysis we applied the novel calibration method [14] which enabled us to estimate the accurate macrostress in the gauge volume located inside the neck. We observed that for advanced necking lattice strains in the austenite were larger than in the ferrite; the phase lattice strains measured at some distance from the neck centre showed a large inversion of the loads localised on both phases. The synchrotron experiment allowed us to study the strain distribution along the neck. For early stages of necking, the difference between lattice strains in the austenite and those in the ferrite evolved more significantly in the neck centre leading to an increase of strains in the austenite and a decrease of strains in the ferrite. It was observed that for the advanced necking process strains in the ferrite decrease significantly apart from the centre (at a distance of about $0.3 \mathrm{~mm}$ ) causing a load transfer to other grains in the sample. Additionally, it was observed that a stress relaxation process occurred in the ferritic grains contributing to 200 and 211 reflections and this conclusion also agrees with the previous neutron experiment [15]. In the work the spatial evolution of the lattice strains along the neck was compared with FEM simulations. The main conclusion in the work was that obtained the equivalent von Mises stress could be responsible for the processes occurring in the phases, especially for the softening (or damage) of particular grains in the ferrite

\section{Acknowledgements}

The work was supported partly by the NCN - Polish National Center for Science, grants: No. UMO - 2011/03/N/ST8/04058 and DEC - 2013/11/B/ST3/03787; and partly by the MNiSW Polish Ministry of Science and Higher Education. We also acknowledge the help offered by the ISIS and ESRF in making their neutron and synchrotron radiation facilities available to us.

\section{REFERENCES}

[1] Bugat, S., Besson, J., Gourgues, A.F., N'Guyen, F. \& Pineau, A. (2001). Microstructure and damage initiation in duplex stainless steel. Mater. Sci. Eng. A 317, 32-36.

[2] Inal, K., Gergaud, P., Francois, M. \& Lebrun, J.L. (1999). X-ray diffraction methodologies of macro and pseudo-macro stress analysis in a textured duplex stainless steel. Scand. $J$. Metall. 28, 139-50.

[3] Baczmański, A., Braham, C. \& Seiler, W. (2003). Microstresses in Textured Polycrystals Studied by Multireflection Diffraction Method and Self Consistent Model. Philosophical Magazine. 83, 3225-3246. 
[4] Jia, N., Cong, Z.H., Sun, X., Cheng, S., Nie, Z.H., Ren. Y., Liaw, P.K., Wang, Y.D. (2009). An in situ high energy X-ray diffraction study of micromechanical behavior of multiple phases in advanced high-strength steels, Acta Mater. 57, 3965-3977.

[5] Neil, C.J., Wollmershauser, J.A., Clausen B., Tomé, C.N. \& Agnew, S.R. (2010). Modeling Lattice Strain Evolution at Finite Strains. Model Verification for Copper and Stainless Steel Using in-situ Diffraction Measurement. Int. J. Plasticity. 26, 1772-1791.

[6] Haušild, P., Berdina, C., Bomparda, P. \& Verdièrec, N. (2001). Ductile fracture of duplex stainless steel with casting defects. Int. J. Pres. Ves. Pip. 78, 607-616.

[7] Balbi, M., Avalos M., Bartali, E. \& Alvarez-Armas, I. (2009). Microcrack growth and fatigue behavior of a duplex stainless steel. Int. J. Fatig. 31, 2006-2013.

[8] Prahl, J., Machová, A., Spielmannová, A., Karlík, M., Landa, M., Haušild, P. \& Lejček, P. (2010). Ductile-brittle behavior at the (110)[001] crack in bcc iron crystals loaded in model. Eng. Fract. Mech. 77, 184-192.

[9] Bunge, H.J. (1982). Texture analysis in material science: Mathematical methods. London: Butterworth.

[10] Daniels, J.E. \& Drakopoulos, M. (2009). High-Energy X-Ray Diffraction Using the Pixium 4700 Flat Panel Detector. Synchrotron Rad. 6, 463-468.

[11] Hammersley, A.P., Svensson, S.O., Hanfland, M., Fitch, S.O. and Häusermann, D. ,1996. Two-dimensional detector software: From real detector to idealised image or two-theta scan. High Pressure Research, 14, 235-248.

[12] Merkel, S. (2011). Multifit/ Polydefix Polycrystal Deformation using X-rays, https://code.google.com/p/multifit-polydefix.

[13] Wroński, S., Baczmański, A., Dakhlaoui, R., Braham, C., Wierzbanowski, K. \& Oliver, E.C. (2007). Determination of Stress Field in Textured Duplex Steel Using TOF Neutron Diffraction Method. Acta Mater. 55, 6219-6233.

[14] Simoms, G. \& Wang, H. (1971). Single Crystal Elastic Constants and Calculated Aggregate Properties: A Handbook, 2nd edition, The M.I.T. Press, Cambridge, Massachusetts and London.

[15] Baczmanski A, Le Joncour L, Panicaud B, et al. (2011). Neutron time-of-flight diffraction used to study aged duplex stainless steel at small and large deformation until sample fracture[J]. Journal of Applied Crystallography. 44(5), 966-982.

[16] Lipinski, P., Berveiller, M., Reubrez, E. \& Morreale, J. (1995). Transition theories of elasticplastic deformation of metallic polycrystals. J. Arch. Appl. Mech. 65, 291-311.

[17] Baczmański, A. \& Braham, C. (2004). Elastoplastic Properties of Duplex Steel Determined Using Neutron Diffraction and Self-Consistent Model. Acta Mater. 52, 1133-1142.

[18] Swift, H.W. (1952). Plastic instability under plane stress, Journal of the Mechanics and Physics of Solids. 1(1), 1-18.

[19] Fréour, S., Gloaguen, D., François, M. \& Guillén, R. (2003). Modelling and simulation of multi-phase effects on X-Ray Elasticity Constants. Phys. Status Solidi (b) 239, 297-309.

[20] Le Joncour L., Panicaud B. \& Baczmański A., et al. (2010). Damage in duplex steels studied at mesoscopic and macroscopic scales [J]. Mechanics of Materials. 42(12), 1048-1063. 